\title{
Time-based Simplified Denavit-Heartenberg Translation (TS- DH) for Capturing Finger Kinematic Data
}

\author{
Mohd Amir Idzham Iberahim¹, Syadiah Nor Wan Shamsuddin ${ }^{1 *}$, Mokhairi Makhtar ${ }^{1}$, Mohd Nordin Abdul Rah- \\ $\operatorname{man}^{1}$, Nordin Simbak ${ }^{2}$ \\ ${ }^{I}$ Faculty of Informatics and Computing, University of Sultan Zainal Abidin (UniSZA), Malaysia \\ ${ }^{2}$ Faculty of Medicine, University of Sultan Zainal Abidin (UniSZA), Malaysia \\ *Corresponding author E-mail: syadiah@unisza.edu.my
}

\begin{abstract}
Virtual finger model is commonly used in many applications for stroke fine motor rehabilitation especially in Virtual Reality (VR) applications. Capturing movement data for fingers is one of the important phases in any virtual fine motor rehabilitation process. Manual observation provides inconsistent evaluation given by different therapists for different rehabilitation sessions. Although the process of capturing data is performed, without time series of captured data, the data will not have a significant impact in producing consistent and meaningful evaluation. Furthermore, the consistency of the assessment of rehabilitation sessions will deliver more robust rehabilitation progress analysis. This data is very useful when paired with time information which can be analyzed to produce optimal evaluation. This paper proposes Time-based Simplified Denavit-Heartenberg Translation (TS-DH) consisting of forward kinematic with simplified DH parameter for capturing coordinate of end of each bone from virtual finger model paired with timeframe data. The DH model is enhanced by implementing 2 additional rules in assigning joint parameter. The data will be recorded with timeframe of every finger movement. As a conclusion, TS-DH model can be used in any virtual finger environment accurately.
\end{abstract}

Keywords: Denavit-Heartenberg (DH) Parameter; Virtual Fine Motor; Forward Kinematic; Stroke Rehabilitation.

\section{Introduction}

Treatments for stroke are different for each patient based on the rate and type of stroke. Stroke treatment can be categorized into three phases; prevention, acute management and rehabilitation as well as secondary prevention [1]. The main goal of treatment is to help stroke patients to develop self-confidence and become as self-sufficient as possible.

Stroke rehabilitation is needed to improve neuro-motor recovery and upper limb impairment by assessing the patient for rehabilitation planning, to observe clinical course and evaluate the rehabilitation result [2]. Common condition of upper limb disability is disorder of motor actions coordination comprising of reaching and grasping [3]. Fine motor especially hand segment loses its motion function easily and the most crucial section to recover in any stroke attack [4].

Hand recovery can be categorized as the main recovery objective because of most of the activities of daily life (ADL) use hand functionality such as feeding, drinking and dressing, and normally if the patient cannot recover their fine motor skills after six months, the chance of recovery is very poor [5].

Hand movement can be shown by transformations of coordinate frame. Many methods were established for representation of kinematic modelling such as Denavit-Hartenberg (DH) notation, Screw theory expression and Lie Algebra. The most famous and broadly used method for kinematic representation is DenavitHartenberg (DH) [6]. DH parameter is needed when kinematic model has open-loop chains and a high number of DOFs [7]. Coordinate of the frame can be found by using forward kinematic.
DH parameters method will define the link and its relations to the next and previous link [8].

The transformation the following four parameters known as DH parameters $[9,10]$ is as follows;

1. $\mathrm{d}$ : displacement along $\mathrm{Z}_{\mathrm{n}-1}$ (distance between link/link offset)

2. $\theta$ : rotation around $Z_{n-1}$ (joint angle)

3. $\mathrm{r}$ or $a$ : displacement along $\mathrm{X}_{\mathrm{n}}$ (link length)

4. $\alpha$ : the angle between $Z_{n}$ and $Z_{n-1}$ around $X_{n}$ (link twist)

DH parameter can be captured in any software application provided that supply parameters that needed. One of the common technology to assist rehabilitation session is Virtual Reality (VR). Stroke rehabilitation technique can gain direct benefit if using VR as assistive technology in pursuit of patient recovery efficiency. A stroke rehabilitation therapist can use VR application as complement method to achieve a better result in post-stroke rehabilitation $[11,12]$ and ease healthcare service burden [13].

In developing any rehabilitation application, the need of constructing a virtual model is very important to capture real hand progress that will be translated into structured data. It will help the application developer to obtain a properly captured parameter to evaluate rehabilitation progress or performance. While most of the recent virtual reality applications for stroke rehabilitation are using automated process to capture progress data, there are still researchers at present who are still using manual observation to evaluate the rehabilitation process [14-17].

Data been captured without proper time sequence will provide only a large set of data with no significant impact. It is important to synchronize data between sequence of collected data in time ordered to provide utility for both data arrangement system and 
the user [18]. Time can be used to capture data trends, recognizing patterns and correlation in the data [19].

This paper is organized as follows. In section 2, the method of assigning DH parameters for finger bone structure is established. In section 3, the proposed model for Time-based Simplified Denavit-Heartenberg Translation (TS-DH) is presented. The experimental results of calculation using TS-DH model by using index finger parameter is also presented in section 4. Finally, our work in this paper is summarized in the last section.

\section{Constructing full transformation matrix from DH parameters}

\subsection{Step 1: Assigning parameters to finger joint}

Based on finger bones structure, all DH parameters will be identified and assigned. There are four rules to be considered when using DH in assigning joint parameter as follows:

1. Z-axis is in direction of the joint axis

2. $\mathrm{X}$-axis is perpendicular to both $Z_{\mathrm{n}}$ and $Z_{\mathrm{n}-1}$

3. Y-axis is in right hand rule

4. $X_{n}$ axis must intersect the $Z_{n-1}$ axis

Proximal Inter-Phalangeal (PIP)

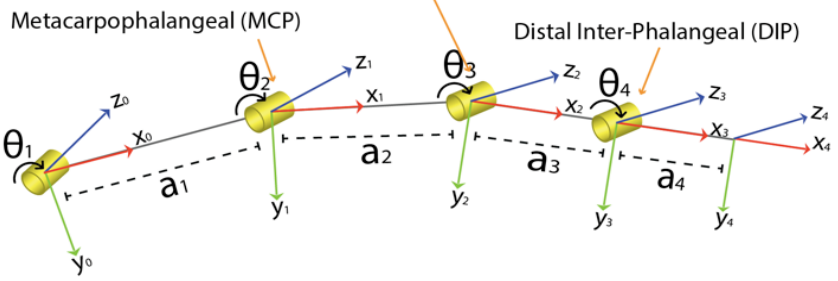

Fig. 1: DH Parameter details by using example of index finger.

Each of joint will be assigned $\boldsymbol{d}$ as distance between link/link offset) along $\boldsymbol{Z}_{\boldsymbol{n - 1}}, \boldsymbol{\theta}$ as joint angle around $\boldsymbol{Z}_{\boldsymbol{n - 1}}, \boldsymbol{r}$ as link length along $\boldsymbol{X}_{\boldsymbol{n}}, \boldsymbol{\alpha}$ as (link twist) around $\boldsymbol{X}_{\boldsymbol{n}}$.

Table 1: DH Parameter by using example of index finger

\begin{tabular}{|c|c|c|c|c|}
\hline Joint (n) & $\boldsymbol{\theta}_{\boldsymbol{n}}$ & $\boldsymbol{\alpha}_{\boldsymbol{n}}$ & $\boldsymbol{d}_{\boldsymbol{n}}$ & $\boldsymbol{r}_{\boldsymbol{n}}$ \\
\hline 1 & $\theta_{1}$ & 0 & 0 & $\boldsymbol{a}_{\mathbf{1}}$ \\
\hline 2 & $\theta_{2}$ & 0 & 0 & $\boldsymbol{a}_{\mathbf{2}}$ \\
\hline 3 & $\theta_{3}$ & 0 & 0 & $\boldsymbol{a}_{\mathbf{3}}$ \\
\hline 4 & $\theta_{4}$ & 0 & 0 & $\boldsymbol{a}_{\mathbf{4}}$ \\
\hline
\end{tabular}

Table 1 shows DH parameters that have been assigned in Figure 1. All 4 DH parameters will be grouped by joint.

\subsection{Step 2: Integration on each parameter into full transformation matrix}

After Table 1 is produced, the parameter will be integrated into transformations matrix. The matrices associated with these operations are two translations and two rotations.

1. Translation matrix for $\boldsymbol{d}$ : distance between link/link offset) along $\boldsymbol{Z}_{n-1}$

Translation $Z_{n-1}\left(d_{n}\right)=\left[\begin{array}{cccc}1 & 0 & 0 & 0 \\ 0 & 1 & 0 & 0 \\ 0 & 0 & 1 & d_{n} \\ 0 & 0 & 0 & 1\end{array}\right]$

2. Rotation matrix for $\theta$ : joint angle around $Z_{n-1}$

Rotation $Z_{n-1}\left(\theta_{n}\right)=\left[\begin{array}{cccc}\cos \left(\theta_{n}\right) & -\sin \left(\theta_{n}\right) & 0 & 0 \\ \sin \left(\theta_{n}\right) & \cos \left(\theta_{n}\right) & 0 & 0 \\ 0 & 0 & 1 & 0 \\ 0 & 0 & 0 & 1\end{array}\right]$
3. Translation matrix for $\boldsymbol{r}$ : link length along $\boldsymbol{X}_{\boldsymbol{n}}$

Translation $X_{n}\left(r_{n}\right)=\left[\begin{array}{cccc}1 & 0 & 0 & r_{n} \\ 0 & 1 & 0 & 0 \\ 0 & 0 & 1 & 0 \\ 0 & 0 & 0 & 1\end{array}\right]$

4. Rotation matrix for $\alpha$ : (link twist) around $\boldsymbol{X}_{\boldsymbol{n}}$

Rotation $X_{n}\left(\alpha_{n}\right)=\left[\begin{array}{cccc}1 & 0 & 0 & 0 \\ 0 & \cos \left(\alpha_{n}\right) & -\sin \left(\alpha_{n}\right) & 0 \\ 0 & \sin \left(\alpha_{n}\right) & \cos \left(\alpha_{n}\right) & 0 \\ 0 & 0 & 0 & 1\end{array}\right]$

Thus, full transformation matrix from Joint $T_{n-1}$ to $T_{n}$ :

$\begin{aligned}{ }^{n-1} T_{n}= & \text { Translation }_{n-1}\left(d_{n}\right) \cdot \text { Rotation }_{n-1}\left(\theta_{n}\right) \\ & \text {. Translation } X_{n}\left(r_{n}\right) \cdot \text { Rotation }_{n}\left(\alpha_{n}\right)\end{aligned}$

$\left[\begin{array}{cccc}\cos \left(\theta_{n}\right) & -\sin \left(\theta_{n}\right) \cdot \cos \left(\alpha_{n}\right) & \sin \left(\theta_{n}\right) \cdot \sin \left(\alpha_{n}\right) & r_{n} \cdot \cos \left(\theta_{n}\right) \\ \sin \left(\theta_{n}\right) & \cos \left(\theta_{n}\right) \cdot \cos \left(\alpha_{n}\right) & -\cos \left(\theta_{n}\right) \cdot \sin \left(\alpha_{n}\right) & r_{n} \cdot \sin \left(\theta_{n}\right) \\ 0 & \sin \left(\alpha_{n}\right) & \cos \left(\alpha_{n}\right) & d_{n} \\ 0 & 0 & 0 & 1\end{array}\right]$

The multiplication operation on each frame matrix to produce representation of end-effector of the finger is

${ }^{0} \mathrm{~T}_{4}={ }^{0} \mathrm{~T}_{1} \cdot{ }^{0} \mathrm{~T}_{2} \cdot{ }^{0} \mathrm{~T}_{3} \cdot{ }^{0} \mathrm{~T}_{4}$

\section{Proposed model for time-based simplified DH translation (TS-DH) model}

This proposed model is constructed from two components; Simplified DH translation model and Time frame block.

\subsection{Simplified DH Translation model}

The model that is represented in virtual model is not strictly bound to real world mechanical design of joint. In virtual model, joints are modelled in a straight line.

Therefore, in assigning parameter in Simplified DH translation model, $d$ and $\alpha$ parameter are constantly valued to zero.

\section{Step 1: Add 2 more rules to normal DH rules}

- $d$ is 0 , no displacement along $Z_{n-1}$

- $\alpha$ is 0 , no the angle between $Z_{n}$ and $Z_{n-1}$ around $X_{n}$.

${ }^{\mathrm{n}-1} \mathrm{~T}_{\mathrm{n}}=\left[\begin{array}{cccc}\cos \left(\theta_{\mathrm{n}}\right) & -\sin \left(\theta_{\mathrm{n}}\right) & 0 & \mathrm{r}_{\mathrm{n}} \cdot \cos \left(\theta_{\mathrm{n}}\right) \\ \sin \left(\theta_{\mathrm{n}}\right) & \cos \left(\theta_{\mathrm{n}}\right) & 0 & \mathrm{r}_{\mathrm{n}} \cdot \sin \left(\theta_{\mathrm{n}}\right) \\ 0 & 0 & 1 & 0 \\ 0 & 0 & 0 & 1\end{array}\right]$

Above is a calculated full transformation matrix with $d$ and $a$ value set to 0 . Full Transformation Matrix can be separated in to two main operations; rotation and translation.

Rotation
$=\left[\begin{array}{ccc}\cos \left(\theta_{n}\right) & -\sin \left(\theta_{n}\right) & 0 \\ \sin \left(\theta_{n}\right) & \cos \left(\theta_{n}\right) & 0 \\ 0 & 0 & 1\end{array}\right]$

Translation $=\left[\begin{array}{c}r_{n} \cdot \cos \left(\theta_{n}\right) \\ r_{n} \cdot \sin \left(\theta_{n}\right) \\ 0\end{array}\right]$ 
Step 2: Each effector for each joint will be measured from wrist and translation data will be used.

${ }^{0} A_{n}$ will represent as transformation matrix from wrist to $\mathrm{n}$ joint meanwhile $x y z_{n}$ will represent as translation of end effector for each joint.

Step 2.1: The measurement from Wrist to $\mathrm{MCP}={ }^{0} A_{1}={ }^{0} T_{1}$

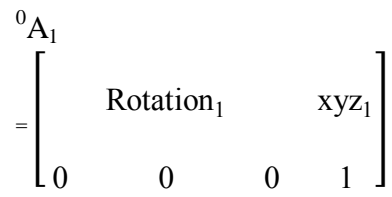

Then, the formula for $\mathrm{X}$ axis translation $x 1=\mathrm{r}_{1} \cdot \cos \left(\theta_{1}\right)$ and formula for $\mathrm{Y}$ axis translation $y 1=\mathrm{r}_{1} \cdot \sin \left(\theta_{1}\right)$ which can be formed as $\mathrm{xyz}_{1}=\left[\begin{array}{c}\mathrm{x} 1 \\ \mathrm{y} 1 \\ 0\end{array}\right]$

Step 2.2: The measurement from Wrist to PIP $={ }^{0} A_{2}={ }^{0} T_{1} \cdot{ }^{1} T_{2}$ ${ }^{0} \mathrm{~A}_{2}$

$\left[\begin{array}{cccc}\text { Rotation }_{2} & & \mathrm{xyz}_{2} \\ 0 & 0 & 0 & 1\end{array}\right]$

Then, the formula for $\mathrm{X}$ axis translation

$\begin{aligned} \mathrm{x} 2= & \left(\left(\cos \left(\theta_{1}\right)\right) \cdot\left(\mathrm{r}_{2} \cdot \cos \left(\theta_{2}\right)\right)\right)+\left(\left(-\sin \left(\theta_{1}\right)\right) \cdot\left(\mathrm{r}_{2} \cdot \sin \left(\theta_{2}\right)\right)\right) \\ & +\left(\left(\mathrm{r}_{1} \cdot \cos \left(\theta_{1}\right)\right)\right)\end{aligned}$

and formula for $\mathrm{Y}$ axis translation

$\mathrm{y} 2=\left(\left(\sin \left(\theta_{1}\right)\right) \cdot\left(\mathrm{r}_{2} \cdot \cos \left(\theta_{2}\right)\right)\right)$

$+\left(\left(\cos \left(\theta_{1}\right)\right) \cdot\left(r_{2} \cdot \sin \left(\theta_{2}\right)\right)\right)+\left(\left(r_{1} \cdot \sin \left(\theta_{1}\right)\right)\right)$

which can be formed as $\boldsymbol{x y z _ { 2 }}=\left[\begin{array}{c}x 2 \\ y 2 \\ 0\end{array}\right]$

Step 2.3: The measurement from Wrist to DIP $={ }^{0} A_{3}=$ ${ }^{0} T_{1} \cdot{ }^{1} T_{2} \cdot{ }^{2} T_{3}$

${ }^{0} \mathrm{~A}_{3}=\left[\begin{array}{cccc} & \text { Rotation }_{3} & & \mathrm{xyz}_{3} \\ 0 & 0 & 0 & 1\end{array}\right]$

Then, the formula for $\mathrm{X}$ axis translation

$$
\begin{aligned}
\mathrm{x} 3= & \left(\left(\left(\left(\cos \left(\theta_{1}\right)\right) \cdot\left(\cos \left(\theta_{2}\right)\right)\right)\right.\right. \\
& \left.\left.+\left(\left(-\sin \left(\theta_{1}\right)\right) \cdot\left(\sin \left(\theta_{2}\right)\right)\right)\right) \cdot\left(\mathrm{r}_{3} \cdot \cos \left(\theta_{3}\right)\right)\right) \\
& +\left(\left(\left(\left(\cos \left(\theta_{1}\right)\right) \cdot\left(-\sin \left(\theta_{2}\right)\right)\right)\right.\right. \\
& \left.\left.+\left(\left(-\sin \left(\theta_{1}\right)\right) \cdot\left(\cos \left(\theta_{2}\right)\right)\right)\right) \cdot\left(\mathrm{r}_{3} \cdot \sin \left(\theta_{3}\right)\right)\right) \\
& +\left(\left(\left(\left(\cos \left(\theta_{1}\right)\right) \cdot\left(\mathrm{r}_{2} \cdot \cos \left(\theta_{2}\right)\right)\right)\right.\right. \\
& +\left(\left(-\sin \left(\theta_{1}\right)\right) \cdot\left(\mathrm{r}_{2} \cdot \sin \left(\theta_{2}\right)\right)\right) \\
& \left.\left.+\left(\left(\mathrm{r}_{1} \cdot \cos \left(\theta_{1}\right)\right)\right)\right)\right)
\end{aligned}
$$

and formula for $\mathrm{Y}$ axis translation

$\mathrm{y} 3=\left(\left(\left(\left(\sin \left(\theta_{1}\right)\right) \cdot\left(\cos \left(\theta_{2}\right)\right)\right)\right.\right.$

$\left.\left.+\left(\left(\cos \left(\theta_{1}\right)\right) \cdot\left(\sin \left(\theta_{2}\right)\right)\right)\right) \cdot\left(\mathrm{r}_{3} \cdot \cos \left(\theta_{3}\right)\right)\right)$

$+\left(\left(\left(\left(\sin \left(\theta_{1}\right)\right) \cdot\left(-\sin \left(\theta_{2}\right)\right)\right)\right.\right.$

$\left.\left.+\left(\left(\cos \left(\theta_{1}\right)\right) \cdot\left(\cos \left(\theta_{2}\right)\right)\right)\right) \cdot\left(r_{3} \cdot \sin \left(\theta_{3}\right)\right)\right)$ $+\left(\left(\left(\left(\sin \left(\theta_{1}\right)\right) \cdot\left(\mathrm{r}_{2} \cdot \cos \left(\theta_{2}\right)\right)\right)\right.\right.$

$+\left(\left(\cos \left(\theta_{1}\right)\right) \cdot\left(\mathrm{r}_{2} \cdot \sin \left(\theta_{2}\right)\right)\right)$

$\left.\left.+\left(\left(\mathrm{r}_{1} \cdot \sin \left(\theta_{1}\right)\right)\right)\right)\right)$

which can be formed as $\boldsymbol{x y z _ { 3 }}=\left[\begin{array}{c}x 3 \\ y 3 \\ 0\end{array}\right]$

Step 2.4: The measurement from Wrist to Fingertip $={ }^{0} A_{4}=$ ${ }^{0} T_{1} \cdot{ }^{1} T_{2} \cdot{ }^{2} T_{3} \cdot{ }^{3} T_{4}$

${ }^{0} \mathrm{~A}_{4}=\left[\begin{array}{cccc} & & & \\ \text { Rotation }_{4} & & \mathrm{xyz}_{4} \\ 0 & 0 & 0 & 1\end{array}\right]$

Then, the formula for $\mathrm{X}$ axis translation

$$
\begin{aligned}
& \left.\mathrm{x} 4=\left(\begin{array}{c}
\left(\left(\left(\left(\cos \left(\theta_{1}\right)\right) \cdot\left(\cos \left(\theta_{2}\right)\right)\right)\right.\right. \\
\left.\left.+\left(\left(-\sin \left(\theta_{1}\right)\right) \cdot\left(\sin \left(\theta_{2}\right)\right)\right)\right) \cdot\left(\cos \left(\theta_{3}\right)\right)\right) \\
+\left(\left(\left(\left(\cos \left(\theta_{1}\right)\right) \cdot\left(-\sin \left(\theta_{2}\right)\right)\right)\right.\right. \\
\left.\left.+\left(\left(-\sin \left(\theta_{1}\right)\right) \cdot\left(\cos \left(\theta_{2}\right)\right)\right)\right) \cdot\left(\sin \left(\theta_{3}\right)\right)\right)
\end{array}\right) \cdot\left(\mathrm{r}_{4} \cdot \cos \left(\theta_{4}\right)\right)\right) \\
& +\left(\left(\begin{array}{c}
\left(\left(\left(\left(\cos \left(\theta_{1}\right)\right) \cdot\left(\cos \left(\theta_{2}\right)\right)\right)\right.\right. \\
\left.\left.+\left(\left(-\sin \left(\theta_{1}\right)\right) \cdot\left(\sin \left(\theta_{2}\right)\right)\right)\right) \cdot\left(-\sin \left(\theta_{3}\right)\right)\right) \\
+\left(\left(\left(\left(\cos \left(\theta_{1}\right)\right) \cdot\left(-\sin \left(\theta_{2}\right)\right)\right)\right.\right. \\
\left.\left.+\left(\left(-\sin \left(\theta_{1}\right)\right) \cdot\left(\cos \left(\theta_{2}\right)\right)\right)\right) \cdot\left(\cos \left(\theta_{3}\right)\right)\right)
\end{array}\right) \cdot\left(\mathrm{r}_{4} \cdot \sin \left(\theta_{4}\right)\right)\right) \\
& +\left(\left(\begin{array}{c}
\left(\left(\left(\left(\cos \left(\theta_{1}\right)\right) \cdot\left(\cos \left(\theta_{2}\right)\right)\right)\right.\right. \\
\left.\left.+\left(\left(-\sin \left(\theta_{1}\right)\right) \cdot\left(\sin \left(\theta_{2}\right)\right)\right)\right) \cdot\left(\mathrm{r}_{3} \cdot \cos \left(\theta_{3}\right)\right)\right) \\
+\left(\left(\left(\left(\cos \left(\theta_{1}\right)\right) \cdot\left(-\sin \left(\theta_{2}\right)\right)\right)\right.\right. \\
\left.\left.+\left(\left(-\sin \left(\theta_{1}\right)\right) \cdot\left(\cos \left(\theta_{2}\right)\right)\right)\right) \cdot\left(\mathrm{r}_{3} \cdot \sin \left(\theta_{3}\right)\right)\right) \\
+\left(\left(\left(\left(\cos \left(\theta_{1}\right)\right) \cdot\left(\mathrm{r}_{2} \cdot \cos \left(\theta_{2}\right)\right)\right)\right.\right. \\
+\left(\left(-\sin \left(\theta_{1}\right)\right) \cdot\left(\mathrm{r}_{2} \cdot \sin \left(\theta_{2}\right)\right)\right) \\
\left.\left.+\left(\left(\mathrm{r}_{1} \cdot \cos \left(\theta_{1}\right)\right)\right)\right)\right)
\end{array}\right)\right)
\end{aligned}
$$

and formula for $\mathrm{Y}$ axis translation

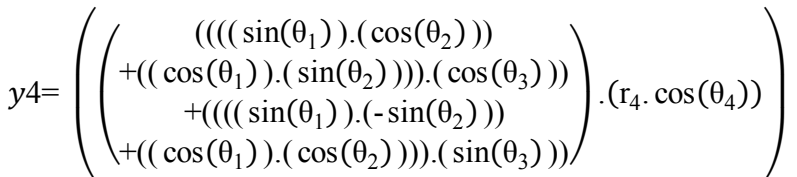

$$
+\left(\left(\begin{array}{c}
\left(\left(\left(\left(\sin \left(\theta_{1}\right)\right) \cdot\left(\cos \left(\theta_{2}\right)\right)\right)\right.\right. \\
\left.\left.+\left(\left(\cos \left(\theta_{1}\right)\right) \cdot\left(\sin \left(\theta_{2}\right)\right)\right)\right) \cdot\left(-\sin \left(\theta_{3}\right)\right)\right) \\
+\left(\left(\left(\left(\sin \left(\theta_{1}\right)\right) \cdot\left(-\sin \left(\theta_{2}\right)\right)\right)\right.\right. \\
\left.\left.+\left(\left(\cos \left(\theta_{1}\right)\right) \cdot\left(\cos \left(\theta_{2}\right)\right)\right)\right) \cdot\left(\cos \left(\theta_{3}\right)\right)\right)
\end{array}\right) \cdot\left(\mathrm{r}_{4} \cdot \sin \left(\theta_{4}\right)\right)\right)
$$

$$
+\left(\left(\begin{array}{c}
\left(\left(\left(\left(\sin \left(\theta_{1}\right)\right) \cdot\left(\cos \left(\theta_{2}\right)\right)\right)\right.\right. \\
\left.\left.+\left(\left(\cos \left(\theta_{1}\right)\right) \cdot\left(\sin \left(\theta_{2}\right)\right)\right)\right) \cdot\left(\mathrm{r}_{3} \cdot \cos \left(\theta_{3}\right)\right)\right) \\
+\left(\left(\left(\left(\sin \left(\theta_{1}\right)\right) \cdot\left(-\sin \left(\theta_{2}\right)\right)\right)+\right.\right. \\
\left.\left.\left(\left(\cos \left(\theta_{1}\right)\right) \cdot\left(\cos \left(\theta_{2}\right)\right)\right)\right) \cdot\left(\mathrm{r}_{3} \cdot \sin \left(\theta_{3}\right)\right)\right)+ \\
\left(\left(\left(\left(\sin \left(\theta_{1}\right)\right) \cdot\left(\mathrm{r}_{2} \cdot \cos \left(\theta_{2}\right)\right)\right)\right.\right. \\
\left.\left.+\left(\left(\cos \left(\theta_{1}\right)\right) \cdot\left(\mathrm{r}_{2} \cdot \sin \left(\theta_{2}\right)\right)\right)+\left(\left(\mathrm{r}_{1} \cdot \sin \left(\theta_{1}\right)\right)\right)\right)\right)
\end{array}\right)\right)
$$

(17) which can be formed as $\boldsymbol{x y z _ { 4 }}=\left[\begin{array}{c}x 4 \\ y 4 \\ 0\end{array}\right]$

\subsection{Time frame block}

Each finger will represent 4 end effectors to each 4-links joint with angle and XYZ axis information for every second. Each finger join will be represented with 4 End-Effector from Link 1 
(Wrist to MCP), Link 2 (Wrist to PIP), Link 3 (Wrist to DIP) and Link 4 (Wrist to Fingertip). Each of Link translation and angle will be recorded based on time frame by finger joint.

\section{Results and discussion}

Let's say we use Wrist to Fingertip measurement calculation for index finger as example $x y z_{4}=\left[\begin{array}{c}x 4 \\ y 4 \\ 0\end{array}\right]$. The example values for link length(r) in milimeter for joint A1 (Wrist to MCP) is $90 \mathrm{~mm}$, joint A2 (MCP to PIP) is $35 \mathrm{~mm}$, joint A3 (PIP to DIP) is $25 \mathrm{~mm}$ and joint A4 (DIP to Fingertip) is $25 \mathrm{~mm}$ (see Table 2). All example values for joint angle $(\theta)$ in degree ${ }^{\circ}$ will be assumed having the same value grouped on time frame. For this example, we will use $5^{\circ}$ and will be converted into radian to be calculated in formula. So, the result will be $x y z_{4}=\left[\begin{array}{c}171.77 \\ 28.94 \\ 0\end{array}\right]$.

Table 2: Collected Data using (TS-DH) Model for Index finger

\begin{tabular}{|c|c|c|c|c|c|}
\hline Joint & $\mathrm{r}(\mathrm{mm})$ & $\theta\left({ }^{\circ}\right)$ & $\mathrm{X}$ axis & $\mathrm{Y}$ axis & $\begin{array}{c}\text { Time Frame } \\
\text { (second) }\end{array}$ \\
\hline $\mathrm{A} 1$ & 90 & 5 & 89.66 & 7.84 & 1 \\
\hline $\mathrm{A} 2$ & 35 & 5 & 124.13 & 13.92 & 1 \\
\hline $\mathrm{A} 3$ & 25 & 5 & 148.27 & 20.39 & 1 \\
\hline $\mathrm{A} 4$ & 25 & 5 & 171.77 & 28.94 & 1 \\
\hline $\mathrm{B} 1$ & 90 & 7 & 89.33 & 10.97 & 2 \\
\hline B2 & 35 & 7 & 123.29 & 19.44 & 2 \\
\hline B3 & 25 & 7 & 146.63 & 28.39 & 2 \\
\hline B4 & 25 & 7 & 168.7 & 40.13 & 2 \\
\hline C1 & 90 & 12 & 88.03 & 18.71 & 3 \\
\hline C2 & 35 & 12 & 120.01 & 32.95 & 3 \\
\hline C3 & 25 & 12 & 140.23 & 47.64 & 3 \\
\hline C4 & 25 & 12 & 156.96 & 66.22 & 3 \\
\hline
\end{tabular}

Table 2 shows TS-DH model result if joint angle added with value of $7^{\circ}$ (for $\mathrm{B} 1$ to $\mathrm{B} 4$ ) and $12^{\circ}$ (for $\mathrm{C} 1$ to $\mathrm{C} 4$ ) paired with time frame for next 2 second reading. Data will be differentiated by finger type and time frame. Every time there is a frame in every link, $X$ and $\mathrm{Y}$ axis will be calculated based on joint angle $(\theta)$ and link length $(r)$ provided by application.

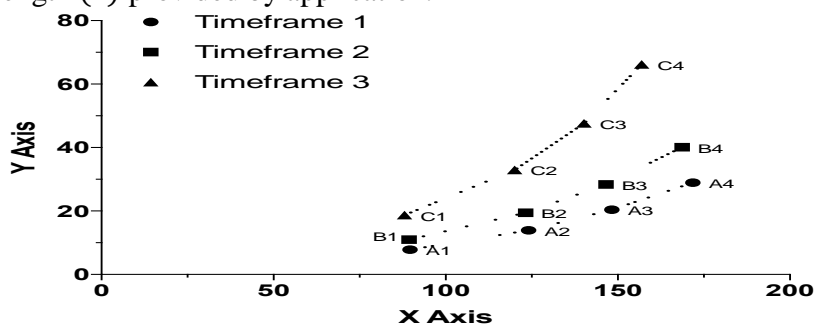

Fig. 2: Four joints of index finger $X Y$ axis movement grouped by timeframe for each three second.

Figure 2 shows the index finger movement of four joint grouped by timeframe 1 to timeframe 3 . A1, A2, A3 and A4 are the joint numbers for timeframe 1. B1, B2, B3 and B4 are the joint numbers for timeframe 2. $\mathrm{C} 1, \mathrm{C} 2, \mathrm{C} 3$ and $\mathrm{C} 4$ are the joint numbers for timeframe 3.

The $\mathrm{Y}$ axis represents vertical finger movement and the $\mathrm{X}$ axis represents horizontal finger movement. The graph can be evaluated as a progress effort for all four joint of index finger by the stroke patient.

\section{Conclusion}

In conclusion, the TS-DH could be a solid model to be implemented in a variety of virtual reality applications with only two main variables needed to be supplied; length of finger bone and joint angle. The result in Figure 2 shows clean and solid coordinate output that can be used to measure rehabilitation progress.
This model could be a standard for virtual reality joint coordination evaluation for fine motor stroke rehabilitation.

In the future, TS-DH could be improved by extending detection from wrist to shoulder. A future study would be needed to explore the use of TS-DH on fine and gross motor combination coordination for upper extremity rehabilitation progress evaluation.

\section{Acknowledgement}

This research was supported in part by e-Science Fund and UniSZA Virtual Reality (VR) grant.

\section{References}

[1] E. Carmeli, S. Peleg, G. Bartur, E. Elbo, and J.-J. Vatine, "HandTutorTM enhanced hand rehabilitation after stroke - a pilot study," Physiother. Res. Int., vol. 16, no. 4, pp. 191-200, Dec. 2011.

[2] A. Scano et al., "Kinect One-based biomechanical assessment of upperlimb performance compared to clinical scales in post-stroke patients," pp. 5720-5723, 2015.

[3] D. Leonardis et al., "An EMG-Controlled Robotic Hand Exoskeleton for Bilateral Rehabilitation," IEEE Trans. Haptics, vol. 8, no. 2, pp. 140-151, Apr. 2015.

[4] S. Guo, F. Zhang, W. Wei, F. Zhao, and Y. Wang, "Kinematic analysis of a novel exoskeleton finger rehabilitation robot for stroke patients," in 2014 IEEE International Conference on Mechatronics and Automation, 2014, pp. 924-929.

[5] D. Zhang, Y. Geng, X. Zhang, Y. Zhang, and G. Li, "Real-time performance of hand motion recognition using kinematic signals for impaired hand function training," Neural Eng. (NER), 2013 6th Int IEEE/EMBS Conf., pp. 339-342, 2013.

[6] X. Huo, Y. Liu, L. Jiang, and H. Liu, "Optimal kinematic control of humanoid arms with offset wrist," in 2014 IEEE International Conference on Mechatronics and Automation, 2014, pp. 1011-1016.

[7] E. Peña-Pitarch, N. T. Falguera, and J. J. Yang, "Virtual human hand: Model and kinematics," Comput. Methods Biomech. Biomed. Engin. vol. 17, no. 5, pp. 568-579, Apr. 2014.

[8] S. Guo, W. Zhang, W. Wei, J. Guo, Y. Ji, and Y. Wang, "A kinematic model of an upper limb rehabilitation robot system," 2013 IEEE Int. Conf. Mechatronics Autom., pp. 968-973, 2013.

[9] J. M. Ibarra Zannatha, A. J. M. Tamayo, Á. D. G. Sánchez, J. E. L. Delgado, L. E. R. Cheu, and W. A. S. Arévalo, "Development of a system based on 3D vision, interactive virtual environments, ergonometric signals and a humanoid for stroke rehabilitation," Comput. Methods Programs Biomed., vol. 112, no. 2, pp. 239-249, Nov. 2013.

[10] G. Du and P. Zhang, "A Markerless Human-Robot Interface Using Particle Filter and Kalman Filter for Dual Robots," IEEE Trans. Ind. Electron., vol. 62, no. 4, pp. 2257-2264, Apr. 2015.

[11] P. Prashun, G. Hadley, C. Gatzidis, and I. Swain, "Investigating the Trend of Virtual Reality-Based Stroke Rehabilitation Systems," in 2010 14th International Conference Information Visualisation, 2010, pp. 641-647.

[12] J. W. Burke, P. J. Morrow, M. D. J. McNeill, S. M. McDonough, and D. K. Charles, "Vision based games for upper-limb stroke rehabilitation," Proc. - IMVIP 2008, 2008 Int. Mach. Vis. Image Process. Conf., pp. $159-164,2008$

[13] J. Island, G. Energy, and G. Energy, "Combined Kinematic Analysis of a Hybrid 7-DOF Rehabilitation,” pp. 1752-1755, 2012.

[14] Y. H. Choi, J. Ku, H. Lim, Y. H. Kim, and N. J. Paik, "Mobile gamebased virtual reality rehabilitation program for upper limb dysfunction after ischemic stroke," Restor. Neurol. Neurosci., vol. 34, no. 3, pp. 455-463, 2016.

[15] K. H. Kong et al., "Efficacy of a virtual reality commercial gaming device in upper limb recovery after stroke: A randomized, controlled study," Top. Stroke Rehabil., vol. 23, no. 5, pp. 333-340, 2016.

[16] D. Kairy et al., "Maximizing post-stroke upper limb rehabilitation using a novel telerehabilitation interactive virtual reality system in the patient's home: Study protocol of a randomized clinical trial," Contemp. Clin. Trials, vol. 47, pp. 49-53, 2016.

[17] J. Kim, M. Lee, Y. Kim, S.-D. Eun, and B. Yoon, "Feasibility of an individually tailored virtual reality program for improving upper motor functions and activities of daily living in chronic stroke survivors: A case series," Eur. J. Integr. Med., vol. 8, no. 5, pp. 731-737, 2016.

[18] T. D. C. Little and A. Ghafoor, "Interval-based conceptual models for time-dependent multimedia data," IEEE Trans. Knowl. Data Eng., vol. 5, no. 4, pp. 551-563, 1993.

[19] A. Rind, T. Lammarsch, W. Aigner, B. Alsallakh, and S. Miksch, "TimeBench: A data model and software library for visual analytics of time-oriented data," IEEE Trans. Vis. Comput. Graph., vol. 19, no. 12, pp. 2247-2256, 2013. 